\title{
Design and characterization of immersion ultrasonic transducers for pulsed regime applications
}

\author{
Francesc Suñol, Diego A. Ochoa, Laura R. Suñé and Jose E. García \\ Department of Physics, Universitat Politècnica de Catalunya -- BarcelonaTech, Barcelona, Spain
}

\begin{abstract}
Ultrasonic transducer design is focused to maximize performance in specific applications, usually leading to a complex design and expensive construction and assembly. With the aim to overcome this drawback, a generalpurpose immersion ultrasonic transducer for pulsed regime applications have been developed. The design of each element of the transducer is described in this paper, wherein materials and geometries for each part have been recommended. A simple theoretical model have been proposed in order to predict the form of the received electric signal in the target transducer. The model is based in the assumption that the piezoelectric element acts as an underdamped oscillator, forced by the acoustic field coming from the propagation medium. Excellent agreement between the experimental measurements and the analytical model is achieved. Electrical impedance measurements reveal negligible differences between the resonance frequency of the active element and that of the assembled transducer. The designed devices have been characterized in water, using two identical transducers placed face to face with changeable orientation. Experimental results show a highly linear response and the generation of a collimated acoustic field. The effects of the thickness of the matching layer on the transmission coefficient have been also studied, resulting in a smooth decrease in the received amplitude, which may significantly lower largescale production costs.
\end{abstract}

\section{KEYWORDS}

ultrasonic transducer; piezoceramic transducer; ultrasonic sensing; transducer design

\section{CONTACT}

Francesc Suñol

Email: francesc.sunol@upc.edu

Telephone: (+34) 934016086

Address: Department of Physics, Universitat Politècnica de Catalunya -- BarcelonaTech, C/Jordi Girona 31, Barcelona 08034, Spain. 


\section{Introduction}

Most of ultrasonic systems use piezoelectric transducers as generators of acoustic energy. In these transducers, the electrical energy supplied by electronic sources is converted into mechanical vibrations as a result of the piezoelectric effect. The active element of a transducer consist in a material with suitable piezoelectric properties since it is the responsible for the energy conversion. In general, piezoelectric materials fall into two categories: hard materials and soft materials. Comparatively, hard materials exhibit smaller piezoelectric constants but higher mechanical quality factors. The combination of properties of hard materials makes them suitable for high-power applications. Soft materials exhibit larger piezoelectric constants, making them ideal for sensing applications. While hard materials are usually excited with a continuous periodic signal, soft materials are generally excited by a single or multiple pulses that are converted to acoustic energy and transferred to the propagation medium. In a similar fashion, ultrasonic transducers are broadly classified into two main groups: narrow-band and broad-band transducers. Narrow-band transducers are frequently used in high-intensity applications, where continuous signals of low frequencies (of the order of $100 \mathrm{kHz}$ ) are predominant. On the other hand, broad-band transducers are generally used in measurement, detection and control applications, using short ultrasonic pulses and frequencies in the range of few $\mathrm{MHz}$.

Concerning the utilization of broad-band transducers, which in general use soft piezo materials, a wide range of potential industrial applications exist. Non-destructive testing, flow metering ${ }^{[1-}$ ${ }^{4]}$, material evaluation ${ }^{[5]}$ and medical diagnosis ${ }^{[6,7]}$ are the major application areas. Furthermore, ultrasonic transducers are widely used for the measurement of diverse quantities, such as detection of interfacial position on two-phase (gas-liquid) flows ${ }^{[8]}$, short range vehicle detection in smart cars ${ }^{[9]}$, or measurement of thickness, position, or fluid properties such as density, viscosity, etc. The measurement principle used in the cited applications usually consist in observing changes in the velocity or the intensity of the ultrasonic wave, and to deduce the 
magnitude of the variable that caused the chang $\mathrm{e}^{[10]}$. The use of the transducers in pulsed regime basically follows two techniques: through-transmission and pulse-echo. In the throughtransmission technique, a transmitter transducer is placed on one side of the test object and a receiver transducer on the other. Ensuring a proper alignment of the transducers, the received ultrasound intensity is monitored. Any defect or impedance change in the path of the ultrasound beam causes a drop in or a complete lack of the received signal, which reveals the presence of the anomaly ${ }^{[10]}$. In the pulse-echo technique a short pulse of ultrasound waves is sent to the object and echoes come back from defects, discontinuities or boundaries.

The basic structure of transducers consists essentially of a piezoelectric active element, usually in form of disc or ring, vibrating in thickness mode, bonded to a backing block on its rear face to damp the vibration, and an acoustic matching layer in the front face to enhance the transmission of the acoustic energy to the propagation medium. A housing is generally used to protect the piezoelectric ceramics from damage and possible corrosion. However, numerous applications require a much more complex (and expensive) transducer structure. The generation of short pulses and a reception of those pulses with good fidelity and sensitivity has a decisive influence in achieving high axial resolution. Furthermore, the perfect transmission of energy is selective and, consequently, influences the spectral characteristics of the transducer. For these reasons, it is evident that transducer design turns out to be a challenging task for certain potential applications.

The main objective of this work is to develop an immersion transducer that is adaptable to a wide range of industrial applications. The design of the transducer is based on using a soft piezoceramic to be operated in pulsed regime applications. The designed device must be versatile, portable, with a low cost and easy fabrication, assembly and replacement. The design of each part of the transducer is firstly described. The measured received signal is obtained and compared with that of a simple theoretical model, based on the assumption that the active element acts as 
an underdamped oscillator forced by the acoustic field. The behavior of assembled transducers are characterized and the generated acoustic field is analyzed.

\section{Transducer design}

An ultrasonic transducer converts electrical energy into mechanical energy, in form of acoustic waves, and viceversa. Figure 1 shows a sketch of the main components of a general transducer. The active element, typically a piezoceramic, is the fundamental part of any transducer, since is the responsible for the energy conversion. The matching layer ensures a maximum transmission of the acoustic wave generated by the active element to the propagation medium. Backing consists in an absorbing material located at the rear face of the active element that controls the vibration of the transducer. Housing or wear plate is the body of the transducer, usually made with rigid material to keep the active element and backing fixed, and protecting all the components from possible corrosions from the working environment. In the next subsections, a detailed description of the design of each component is presented.

\section{Active element}

Transducer performance depends on its internal structure and on constitutive material properties, the most important of which is the active piezoelectric element. The classical active element, in particular for medical imaging and flow measurement applications, is based on a piezoelectric plate or disc poled along the thickness direction, whose thickness defines the resonance frequency of the device.

Active elements can be piezoceramic, monolithic single crystal, piezopolymer or piezocomposite materials. Due to its low production costs, piezoceramic materials prevail in the market worldwide. The most common piezoceramic material used as active material in ultrasonic transducers is lead zirconate titanate (PZT), as it has a high electromechanical coupling 
coefficient and a high dielectric constant. In addition to its good electromechanical properties, it allows good electrical matching to cables and electronics (which are typically at 50 to $80 \Omega^{[11]}$ ). PZT-based transducers are currently widely used for non-destructive testing due to their small size, low cost, and their ability to work both as actuator or sensor.

The main disadvantages of PZT are its large acoustic impedance of approximately 33 MRayl (compared to water or tissue, which are around 1.5 MRayl) and that it contains lead, which is toxic. On one hand, PZT piezoceramics are typically used in numerous applications because of their high coupling factor, even through their acoustic impedance is high. No material have been discovered that allows to obtain both high coupling and acoustic impedance similar to that of water or biological tissues. Nevertheless, the mismatch in acoustic impedance can be compensated by using additional layers in the transducer structures. On the other hand, lead-free alternatives aiming at replacing the market-dominant materials have been extensively searched for more than a decade worldwide ${ }^{[12-14]}$. To date, some commercial outputs for specific applications have been reported (such as barium strontium zirconate titanate --BSZT--, potasium sodium niobate --KNN-- or sodium bismuth titanate --BNT-- based materials $\left.{ }^{[15,16]}\right)$, but the invention of a lead-free piezoceramic with a performance comparable to that of PZT does not seem to be fulfilled yet ${ }^{[17]}$. Other alternatives to PZT such as new-generation lead zinc niobatelead titanate (PZN-PT) or lead magnesium niobate-lead titanate (PMN-PT) offer excellent piezoelectric properties $^{[18]}$, however the manufacturing costs are much higher than that of PZT. Considering these requirements, a soft PZT material (APC 850) is selected in this work as good candidate to work in pulsed regime. The physical properties of the used piezoelectric ceramics are: density $\rho=7600 \mathrm{~kg} / \mathrm{m}^{3}$; piezoelectric charge coefficient $d_{33}=400 \mathrm{pC} / \mathrm{N}$, Curie temperature $T_{\mathrm{C}}=360{ }^{\circ} \mathrm{C}$, and mechanical quality factor $\mathrm{Q}=80$. The selected geometry of the active element is a disc with a diameter of $a=1 \mathrm{~cm}$ and a thickness of $h=1 \mathrm{~mm}$. The thickness-mode resonance frequencies of a cylindrically-shaped object are 


$$
f_{s}=\frac{c}{2} \frac{p}{h}
$$

where $p=1,2,3, \ldots$ and $c=4350 \mathrm{~m} / \mathrm{s}$ is the speed of sound in the material. Therefore, the fundamental resonance frequency in thickness mode is around $2 \mathrm{MHz}$. This frequency is adequate for fluid flow metering and medical diagnosis applications, due to a low wave dispersion in the propagation medium. With the aim to facilitate the construction of the transducer, silver wraparound electrodes have been chosen. Within the wrap-around configuration, the external electrode extends to the internal face of the active element, allowing to solder both electrodes in the internal face thus providing a flat surface for an undistorted propagation the acoustic waves.

\section{Matching layer}

The active element has an acoustic impedance much higher than that of water or biological tissues. This large difference leads to an acoustical mismatch, that unearth the need of including additional layers for improving the energy transmission. The thickness of a matching layer is generally around a quarter-wavelength at the resonance frequency, and its acoustical impedance is intermediate between those of the piezoceramic and the propagation medium. The quarter wavelength matching layer allows maximum transmission for a single frequency of an ultrasound wave ${ }^{[19]}$. However, pulsed wave systems produce ultrasound waves across a range of frequencies, making the optimal single matching layer difficult to design. The use of multiple matching layers can further improve transducer performance, at the expense of adding more complexity in the design and fabrication processes ${ }^{[20,21]}$.

The simplest transmission system is comprised of an active element $a$ and a propagation medium $m$. A plane ultrasonic wave entering on a straight boundary between two media will both reflect back and transmit to the adjacent medium. The pressure amplitudes of the reflected and transmitted waves are controlled by the acoustic impedance ratio of the two media. Specific acoustic impedance $Z$ is given by $Z=\rho c$, where $\rho$ is the density and $c$ is the acoustic velocity of 
the medium. The expressions for the reflectance $R$ and transmittance $T$ for an incoming wave from the active element are ${ }^{[22]}$

and

$$
R=\left(\frac{Z_{m}-Z_{a}}{Z_{m}+Z_{a}}\right)^{2}
$$

$$
T=\frac{4 Z_{m} Z_{a}}{\left(Z_{m}+Z_{a}\right)^{2}}
$$

respectively, where $Z_{a}$ and $Z_{m}$ are specific acoustic impedance of the active element and the propagation medium, respectively. When $Z_{m}$ is considerably smaller than $Z_{a}$, which is the case of a piezoceramic active element and a water or tissue loading medium, the reflectance approaches 1 and the transmittance approaches 0 . Therefore, in order to improve the wave transmission to the loading medium, the addition of an acoustic matching layer becomes imperative.

The acoustic impedance of the matching layer has an intermediate value between the active piezoelectric material and the loading medium, and its specific value is essential for reducing the reflection of the ultrasonic wave. When an intermediate layer $\ell$ (with thickness $d_{\ell}$ and a speed of sound $c_{\ell}$ ) is inserted between media $a$ and $m$, the transmittance of acoustic intensity from medium $a$ to $m$ can be described as a function of $Z_{a}, Z_{m}$ and $Z_{\ell}$. By introducing the dimensionless parameters $\xi=Z_{a} / Z_{m}$ and $\phi=Z_{\ell} / s q r t\left\{Z_{-} a Z_{-} m\right\}$, the transmittance is found to be simply expressed $\mathrm{as}^{[19,22]}$

where

$$
T=T_{0}\left[\cos ^{2}\left(\frac{2 \pi f}{c_{\ell}}\right) d_{\ell}+A \sin ^{2}\left(\frac{2 \pi f}{c_{\ell}}\right) d_{\ell}\right]^{-1},
$$

$$
A=\frac{\xi\left(1+\phi^{2}\right)^{2}}{[\phi(1+\xi)]^{2}}
$$

and

$$
\mathrm{T}_{0}=\frac{4 \xi}{(1+\xi)^{2}}
$$


$T_{0}$ is the transmittance without matching layer and is independent of $f$. At the resonance frequency $f_{s}$, the ratio $\varphi=T / T_{0}$ has its maximum value $\varphi_{s}=A^{-1}$. The value of $f_{s}$ is governed by $d_{\ell}$ and $c \ell$, and the value of $\varphi_{s}$ is determined by $Z_{a}, Z_{m}$ and $Z_{\ell}$.

Theoretically, $100 \%$ transmission is shown to occur for a sinusoidal wave when both conditions

$$
\mathrm{Z}_{\ell}=\sqrt{\mathrm{Z}_{\mathrm{a}} \mathrm{Z}_{\mathrm{m}}}
$$

and

$$
d_{\ell}=c_{\ell} /(4 f)
$$

are met (for a wideband signal, the acoustic impedance of a single matching layer should be modified to $\left.Z_{\ell}=\left(Z_{a} Z_{m}^{2}\right)^{1 / 3}\right)$. Under these circumstances, the thickness of the matching layer is a quarter-wavelength of the acoustic wave, $d_{\ell} \$ \mathrm{~d}_{-} \mid \mathrm{ell}=\lambda \ell / 4$, hence $T=1$ and the ultrasonic wave enters the medium $m$ without losses.

A good candidate material to be used as a matching layer with reasonable performance turns out to be the bi-component epoxy resin Weicon MS- $1000^{\circledR}$. The acoustic impedance of this material can be computed from the product between the density and the sound speed in the medium, which can be approximated as

$$
c=\sqrt{\frac{K+\frac{4}{3} G}{\rho}}=\sqrt{\frac{E(1-v)}{\rho(1+v)(1-2 v)}}
$$

where $K, G, E$ and $v$ are the bulk modulus, shear modulus, Young modulus and the Poisson ratio, respectively. With this, the acoustic impedance of the epoxy resin is $Z_{\ell}=4.3 \mathrm{MRayl}$, a value in between the theoretical prediction by Equation (7) and the ideal value for a wideband transducer if one considers water as the propagation medium.

In practice, $100 \%$ transmission is impossible for only considering the front matching layer. Due to the acoustic mismatch between the loading medium and the piezoelectric material, a reflected wave reverberates inside the transducer element. This would cause long ring-down of the ultrasonic pulse, which is the so-called ringing effect ${ }^{[23]}$. In some applications (i.e. diagnostic 
imaging), it is highly undesirable to have a pulse with long duration. A backing layer can be used to damp out the ringing by absorbing part of the energy from the vibration of the back face.

\section{Backing material}

The main problem with transducers used for numerous applications is the large acoustic mismatch between the active element and the load (e.g. water, tissue), resulting in most of the acoustic energy being reflected back and forth between the front and rear faces of the element. Consequently, the transducer sends a pulse with long duration resulting in low axial resolution. These oscillations may be dampened by positioning a lossy backing material behind the piezoelectric element to absorb a large proportion of the energy. The consequence is a lower amplitude transmitted signal, but a higher axial resolution. Therefore, the addition of a backing layer increases the bandwidth of the transducer, at the expense of reducing its sensitivity. Thus, a trade-off has to be performed for each application. If there is a mismatch in acoustic impedance between the active element and the backing material, more energy will be reflected forward into the propagation medium. As a result, the transducer has lower resolution due to a longer waveform duration, but it has greater sensitivity due to higher signal amplitude.

The present work is focused on the development of an immersion device that is capable to transmit as much energy as possible. This is reflected in a higher signal amplitude received in the target transducer. For this reason, the design of the transducer stands on a backing material that has a high difference in acoustic impedances with respect that of the active element. As a consequence, air is suggested as a backing material that results in a reasonably high sensitivity and efficiency transducer. Air-backed transducers have been developed for many decades. It was found that air-backing devices could be constructed without affecting the transducer bandwidth considerably ${ }^{[24,25]}$. With the aim to construct a transducer with air as a backing material without 
losing mechanical stability, a housing with an annular support have been designed, leaving an air-filled region in form of paraboloid.

\section{Housing}

The basic purpose of the housing is to protect the transducer elements from the testing environment. In the case of contact or immersion transducers, the housing must be a durable and corrosion-resistant material in order to withstand the wear caused by use on other materials. Concerning the geometry of the housing, it should permit easy assembly and it should resist highpressures (e.g. transducers in flow metering applications generally withstand pressures around 16 bars or higher). A shape with axial symmetry becomes convenient, due to its versatility and ease of fabrication. At the same time, the transducer should be designed with the aim to allow a maximum energy transmission to the propagation medium.

The body of the transducer should be solid and robust while it should be simple to build, with a reasonably low cost material. Adequate materials for the body of immersion transducers are polyvinyl chloride (PVC) or Polipropilene. In Figure 2 (left), a scheme of the proposed housing is presented. The air backing is located inside a paraboloid region, with its focal point placed at the center of the active element, described by the equation $y=-x^{2} / 8+2$, being $y$ the axial symmetry axis and $x$ the radial axis. This geometry has the peculiarity that the acoustic waves coming from the active element are reflected in the back face of the backing region and directed back to the active element with normal incidence to its shape. Even if the influence of the reflected wave is negligible on the operation of the transducer, a paraboloid shape is the optimal (among all other possible shapes) to maximize the energy transmission to the propagation medium. Therefore, the paraboloid geometry have been chosen for the backing-housing interface. To ensure the tightness and robustness of the transducer, an hybrid polymer (Weicon Flex+Bond ${ }^{\circledR}$ ) is used to protect the 
welding and cables. Figure 2 (right) shows pictures of the active element with wrap-around electrodes (the flat external face and the welding internal face) and the assembled transducer.

\section{Transducer characterization}

The designed immersion transducer consists in an air-backed PZT active element with an epoxy resin matching layer, placed inside an axisymmetrical housing. The characterization of the transducers response and the generated acoustic field have been performed in tap water, using two identical transducers as test samples. Unless stated the contrary, all the data concerning peakto-peak response amplitude have been obtained by applying an automated average of $N=512$ measurements.

In Figure 3, a sketch of the measurement setup configuration along with the key parameters used in this work are presented. A pulse of a predefined duration will be applied to the transmitter transducer, with an amplitude provided by an external battery. The electric pulse will be converted to mechanical vibrations of the active element, that propagates through the matching layer and the loading medium in form of acoustic waves. The ultrasonic waves will be received by the target transducer and converted back to an electrical signal, which will have a determined form with a certain amplitude and frequency.

\section{Modeling the form of the received pulse}

With the objective to determine the form of the reference signal, a simple model is proposed. Assuming that the piezoelectric material act as an underdamped oscillator, the reference signal is defined as the solution of a forced oscillator, in which the external force is the acoustic wave received through the propagation medium. If the signal sent to the transmitter transducer is a rectangular pulse $U(t)$, with amplitude $A_{\text {pulse }}$ and width $t_{w}$, the form of the acoustic signal is given by the solution of the non-homogeneous differential equation 


$$
\ddot{\mathrm{X}}_{a s}+2 \xi \omega_{n} \dot{\mathrm{X}}_{a s}+\omega_{n}^{2} X_{a s}=U(t)
$$

where the subscript as stands for "acoustic signal”, $\omega_{n}=2 \pi f_{n}$ with $f_{n}$ the natural frequency of the piezoelectric material, $\xi$ is the damping coefficient and $U(t)$ is the electric signal sent to the transmitter transducer. $U(t)$ can be written as $U(t)=A_{\text {pulse }}\left[\Theta(t)-\Theta\left(t-t_{w}\right)\right]$, where $\Theta(t)$ is the Heaviside step function. A transient particular solution of Equation (10) can be written $\operatorname{as}^{[26]}$

$$
\mathrm{X}_{\mathrm{as}}(\mathrm{t})=\mathrm{A}_{\text {pulse }}\left[\mathrm{g}(\mathrm{t})-\mathrm{g}\left(\mathrm{t}-\mathrm{t}_{\mathrm{w}}\right)\right]
$$

where

$$
g(t)=\left[1-e^{-a t}\left(\cos (b t)+\frac{a}{b} \sin (b t)\right)\right] \Theta(t),
$$

with $a=\xi \omega_{n}$ and $b=\omega_{n} \backslash \operatorname{sqrt}\left\{1-\backslash \mathrm{xi}^{\wedge} 2\right\}$. Equation (11) represents the acoustic signal that travels through the propagation medium, and is received by the target transducer. Since the transmitter and receiver transducers are identical, the form of the electric signal induced in the receiver transducer will be the solution of an underdamped oscillator (with the same physical parameters as the transmitter transducer), under an external force driven by the acoustic signal given by Equation (11). That is,

$$
\ddot{\mathrm{X}}+2 \xi \omega_{n} \dot{\mathrm{X}}+\omega_{n}^{2} X=X_{a s}(t) \text {. }
$$

Therefore, the solution of Equation (13) will resemble the electric signal provided by the receiver transducer, and will be designated as the reference signal. The analytical solution of Equation (13) is detailed in Appendix A and is given by

$$
X(t)=\frac{A_{\text {pulse }}}{b}\left[T_{1}(t)-T_{2}(t)\right] \Theta(t)
$$

In order to determine the validity of this approach, the solution (Equation (14)) has been plotted and compared with the received electric signal. The presented model takes four independent parameters as input variables, namely $A$ (pulse amplitude), $t_{w}$ (pulse width), $f_{n}$ (transducer resonance frequency) and $\xi$ (damping coefficient). Numerical values of these parameters have 
been set according to the transducer and electric pulse characteristics. A moving-average filter ${ }^{[27]}$ have been implemented with the aim to visualize the form of the received signal without noise disturbances. The filtered signal at time $t_{i}$ is obtained by

$$
\mathrm{Y}\left(\mathrm{t}_{\mathrm{i}}\right)=\frac{1}{\mathrm{~N}_{M A}} \sum_{\mathrm{j}=-\mathrm{N}_{M A} / 2}^{\mathrm{N}_{M A} / 2} \mathrm{Y}_{\mathrm{n}}\left(\mathrm{t}_{\mathrm{i}}+\mathrm{t}_{\mathrm{j}}\right)
$$

where $Y_{n}$ is the non-filtered signal received by the transducer, and $N_{\mathrm{MA}}$ is the number of points used to compute the moving average filter. A comparison between the received signal $Y(t)$ obtained experimentally using $N_{\mathrm{MA}}=100$, and the prediction by the proposed model given by Equation (14), is presented in Figure 4. Excellent agreement is achieved, despite the simplicity of the model. This not easy sincemany ultrasound transducers have been designed for medical purposes $^{[28,29]}$, but could not obtain such a good agreement using an RLC electrical circuit model.

\section{Electrical impedance characterization}

By applying an electric pulse with a determined duration, the efficiency of the energy conversion can be maximized if the correct value of the pulse duration is used. The optimal value is determined by the resonance frequency of the transducer. Even though the resonance frequency of the active element can be determined given its geometrical and physical properties (Equation (1)), the determination of the resonance frequency of the whole transducer from fundamental concepts becomes non-viable due to its different material composition and geometrical complexity. An appropriate way to determine precisely the resonance frequency of the transducer is to search for minima of the electric impedance for different frequencies, with a phase shift close to $\Delta \varphi \approx 180^{\circ}$. The minima in electric impedance correspond to resonance frequencies, while the maxima corresponds to anti-resonances.

The typical behavior of the electrical impedance of piezoelectric transducers in the resonance neighborhood is characterized by abrupt variations. Consequently, small shifting of the applied 
frequency translates to large variations in the electrical behavior of the transducer. These variations usually lead to mismatching between the transducer and the electronic driving circuit. As a consequence, the performance of the system is affected, the vibration amplitude decreases, as well as the energy conversion rate and the acoustic energy on the radiated media ${ }^{[30]}$.

By applying a frequency sweep using an impedance analyzer (Agilent 4294A), the electrical behavior of both the isolated active element and the assembled transducer have been characterized. Results are presented in Figure 5. It can be observed (Figure 5 (left)) that at $f \approx 2$ $\mathrm{MHz}$, there is a local minimum in electric impedance together with a phase shift of $\Delta \varphi \approx 170^{\circ}$, which is an indicative of the quality of the active element. Satellite peaks appearing in Figure 5 (left) show the coupling between the thickness extensional mode and lower frequency modes (radial modes). However, these smaller peaks are not associated to a considerable phase shift. When the active element is assembled into the transducer (Figure 5 (right)), the minimum of electric impedance presents a smoother and inevitably deteriorated curve (radial modes of the active element are clamped due to the housing) and the resonance frequency is found to be located at $f_{s}=2.02 \mathrm{MHz}$. In the present case, the exact location of the resonance frequencies of the isolated active element and the assembled transducer can be considered the same in practical terms.

\section{Geometrical and resonance effects}

The resonance frequency of the transducer determines the appropriate value of the pulse duration sent to the transmitter, which is crucial to excite the transducer effectively. Therefore, as a first consideration it is convenient to measure the effects of the input pulse duration $\left(t_{w}\right)$ on the generated ultrasonic signal. A pulse with

$$
\mathrm{t}_{\mathrm{w}}=\frac{2 \mathrm{n}+1}{2 \mathrm{f}_{\mathrm{s}}}
$$

where $n=0,1,2,3, \ldots$ correspond to the different harmonics, should excite the transducer effectively. Therefore, for the fundamental mode with $n=0$, a pulse duration of $t_{w}=T / 2=1 /\left(2 f_{s}\right) \approx 250 \mathrm{~ns}$ should produce a signal with maximum amplitude. In Figure 6 (left), the 
peak-to-peak amplitude, measured in the receiver transducer, as a function of the pulse duration is presented. The maximum amplitude is obtained using a pulse of $t_{w}=260 \mathrm{~ns}$, very similar to the prediction of $250 \mathrm{~ns}$, hence this is the optimum value to be used in the transducer potential applications. It can also be observed the existence of different maxima, the position of which are correctly determined by Equation (16).

It is also important to know the degree of linearity of the transducer response, with respect the amplitude of the electric pulse applied to the transmitter. With this objective, the peak-to-peak voltage amplitude $\left(A_{p p}\right)$ of the response signal have been measured for different values of the input pulse voltage. Results are presented in Figure 6 (right), where a linear response is observed. The data was obtained with an orientation angle of $\theta=0^{\circ}$, and a separation of $r=10 \mathrm{~cm}$ between transducers, using $t_{\mathrm{w}}=260 \mathrm{~ns}$ and $d_{\ell}=0.5 \mathrm{~mm}$.

In order to characterize the spatial variation of the acoustic field generated by the transmitter transducer, a distance and angle sweep have been performed by moving the receiver transducer with respect to the transmitter transducer, located at a fixed position. The peak-to-peak amplitude of the receiver transducer is plotted as a function of the distance between the transmitter and receiver (for different orientation angles) in Figure 7 (left). It can be noted that there is a slight decrease in amplitude at short distances $(r<4 \mathrm{~cm})$, that could be due to the existence of a near field effect. The near field is a region directly in front of the transducer where the amplitude goes through a series of maxima and minima before stabilizing, due to the interference between waves coming from different parts of the active element. Despite the fact that some authors consider that the existence of the near field only makes sense when applying a continuous wave (thus is not applicable to pulsed regime), in the present case the near field effect recovers it sense due to the nature of the backing, that results in a long ringing effect from which different wavefronts can produce interference. The range of the near field ends at the last maximum, at a distance $d_{n}$ 
from the transducer that, if using an active element in form of a circular disc ${ }^{[31-33]}$, can be computed as

$$
d_{n}=\frac{D^{2} f_{s}}{4 c}
$$

being $D$ its diameter, $f_{s}$ the resonance frequency and $c$ the sound speed at the propagation medium. In the present case, $D=1 \mathrm{~cm}, f_{s}=2 \mathrm{MHz}$ and $c=1480 \mathrm{~m} / \mathrm{s}$, leading to $d_{n} \approx 3.4 \mathrm{~cm}$, in accordance with Figure 7 (left).

Nevertheless, it can be observed that the amplitude does not suffer a considerable decrease along the radial direction in the range of distances considered, which indicates that the acoustic field must be notably collimated. In this way, separating the transducers a few centimeters should not result in a considerable energy loss, as long as the transducers are kept properly aligned.

In Figure 7 (right), the peak-to-peak amplitude is plotted and as a function of the orientation angle (for different distances). In this case, the amplitude has a great dependence on the orientation angle: the transducers should be aligned face to face for an acceptable transmission of the ultrasonic pulse. The opposite case, at orientation angles higher than $\theta=5^{\circ}$ approximately, could lead to a considerable loss of amplitude which can be critical for some applications.

The effects of the thickness of the matching layer, $d \ell$, can be observed in Figure 8, in which the peak-to-peak amplitude is plotted as a function of $d_{\ell}$. A thick matching layer of $d_{\ell} \approx 3.5 \mathrm{~mm}$ was deposited in a pair of transducers, and it was lowered carefully until its optimal value of $d_{\ell}=0.45$ $\mathrm{mm}$. The amplitude received decreases smoothly as the thickness of the matching layer increases, and it reduces abruptly for thickness values of $d_{\ell}>3 \mathrm{~mm}$. This behavior differs from the predicted by Equation (4), since $T$ should appear as a periodic function of $d_{c}$ with a periodicity of $0.9 \mathrm{~mm}$ approximately. Experimentally, such periodicity was not observed. In the light of these results, one can conclude that in the proposed design the matching layer thickness is not critical below a 
$3 \mathrm{~mm}$ range, which may result in a significantly lower large-scale production costs of the transducers.

\section{Conclusions}

A general-purpose immersion transducer for pulsed regime applications have been designed, assembled and tested. The design of each element of the transducer have been described in this study, whereas materials used, and shape geometry for each part have been proposed. A simple model have been developed with the aim to predict the behavior and the shape of the obtained electric signal from the target transducer. In this model, the transmitter and receiver are approximated as underdamped oscillators, the first being forced by a rectangular pulse to generate the ultrasonic wave, while the latter being forced by the acoustic field coming from the propagation medium to produce the electric output signal. Excellent agreement between the experimental measurements and the analytical results are achieved, despite the simplicity of the model proposed. The resonance frequency of the active element and the assembled transducer have been measured using an electric impedance analyzer, revealing that the difference between resonance frequencies is negligible. Assembled transducers have been characterized in water, exhibiting a highly linear response under the $10 \mathrm{~V}$ range of input voltage. The effects of the input pulse duration have also been studied, resulting in a periodic behavior in accordance with the theoretical prediction. With the aim to know the spatial range of the generated acoustic field, two transducers have been placed face to face, in a setup configuration allowing a changeable distance and orientation. A collimated field with an aperture angle less than $5^{\circ}$ have been measured. Finally, the effects of the matching layer thickness have been also characterized, presenting a smooth decrease as the matching layer thickness increase, despite the fact that the theoretical model predicts that the maximums in transmission should exhibit periodicity. In the presented design, the matching layer thickness does not have remarkably effects on the transmission 
coefficient, as long as it kept within a $3 \mathrm{~mm}$ range, which can result in a considerably ease of fabrication thus lowering large-scale production costs. The transducers proposed in this paper are therefore promising candidates to be used in immersion pulsed regime applications, such as liquid flow metering, liquid level sensors or level detectors.

\section{Conflicts of interest}

The authors declare no conflict of interest. The funding sponsors had no role in the design of the study; in the collection, analyses, or interpretation of data; in the writing of the manuscript, and in the decision to publish the results.

\section{Funding}

This work was financially supported by MINECO (Spanish Government), project ESP201572277-EXP.

\section{Author contributions}

Francesc $\boldsymbol{S}$. contributed in the design, modeling and assembly of the transducer, performed experimental measurements, data analysis, and wrote the paper.

Diego A. O. contributed significantly to the design and fabrication of the transducers, and provided helpful scientific support.

Laura R. S. assembled the transducers and performed experimental measurements.

Jose E. G. have supervised this work, which included participating in discussions, planning and designing the experiments and refining the manuscript. 


\section{Appendix A. Analytical solution of the acoustically-forced underdamped oscillator model}

The electric signal provided by the receiver transducer is the solution of the non-homogeneous differential equation

$$
\ddot{\mathrm{X}}+2 \xi \omega_{n} \dot{\mathrm{X}}+\omega_{n}^{2} X=X_{a s}(t)
$$

where $X_{a s}(t)$ is the form of the acoustic signal travelling through the flowing medium,

$$
X_{a s}(t)=A_{\text {pulse }}\left[g(t)-g\left(t-t_{w}\right)\right]
$$

with

$$
g(t)=\left[1-e^{-a t}\left(\cos (b t)+\frac{a}{b} \sin (b t)\right)\right] \Theta(t),
$$

being $a=\xi \omega_{n}, b=\omega_{n} \sqrt{1-\xi^{2}}$ and $\Theta(t)$ the Heaviside step function.

Fortunately, an analytical solution of Equation (18) exists and can be obtained with the aid of the Green function,

$$
\mathrm{X}(\mathrm{t})=\frac{\mathrm{A}_{\text {pulse }}}{\mathrm{b}} \int_{-\infty}^{\mathrm{t}}\left[\mathrm{g}(\tau)-\mathrm{g}\left(\tau-\mathrm{t}_{\mathrm{w}}\right)\right] e^{-a(t-\tau)} \sin [b(t-\tau)] d \tau .
$$

Expanding the integral in two parts,

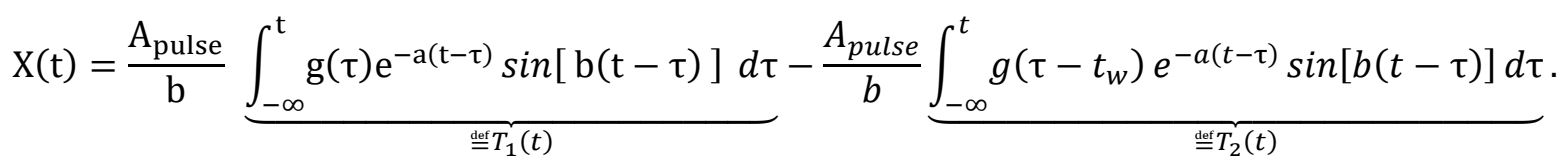

The first term on the right-hand side of Equation (22) is designated as $T_{1}(t)$, whereas the second term is denoted by $T_{2}(t)$. The Heaviside step function contained in $g(t)$ transforms the integration limits from $(-\infty, t]$ to $[0, t]$. Therefore, the term $T_{1}(t)$ can be rewritten as

$$
\mathrm{T}_{1}(\mathrm{t})=\int_{0}^{\mathrm{t}}\left[1-\mathrm{e}^{-\mathrm{a} \tau}\left(\cos (\mathrm{b} \tau)+\frac{\mathrm{a}}{\mathrm{b}} \sin (\mathrm{b} \tau)\right)\right] e^{-a(t-\tau)} \sin [b(t-\tau)] d \tau .
$$

Expanding the three terms in the parenthesis,

$$
\mathrm{T}_{1}(\mathrm{t})=\int_{0}^{\mathrm{t}} \mathrm{e}^{-\mathrm{a}(\mathrm{t}-\tau)} \sin [\mathrm{b}(\mathrm{t}-\tau)] \mathrm{d} \tau-e^{-a t} \int_{0}^{t} \cos (b \tau) \sin [b(t-\tau)] d \tau-
$$




$$
-\frac{a e^{-a t}}{b} \int_{0}^{t} \sin (b \tau) \sin [b(t-\tau)] d \tau
$$

Integrating the three terms separately and rearranging, one obtains

$$
\mathrm{T}_{1}(\mathrm{t})=\mathrm{e}^{-\mathrm{at}}\left[\frac{\mathrm{b} \mathrm{e}^{\mathrm{at}}-\mathrm{a} \sin (\mathrm{bt})-\mathrm{b} \cos (\mathrm{bt})}{\mathrm{a}^{2}+\mathrm{b}^{2}}\right]-e^{-a t}\left[\frac{t \sin (b t)}{2}-\frac{a[\sin (b t)-b t \cos (b t)]}{2 b^{2}}\right] .
$$

Following a similar procedure for the term $T_{2}(t)$, one arrives at

$$
\begin{gathered}
\mathrm{T}_{2}(\mathrm{t})=\frac{\mathrm{e}^{-\mathrm{at}}\left[\mathrm{be} \mathrm{at}^{\mathrm{at}}-\mathrm{a} \sin (\mathrm{bt})-\mathrm{b} \cos (\mathrm{bt})\right]}{a^{2}+b^{2}}-e^{-a\left(t-t_{w}\right)} . \\
. \frac{2 b t \sin \left[b\left(t-t_{w}\right)\right]+\cos \left[b\left(t_{w}-t\right)\right]-\cos \left[b\left(t_{w}+t\right)\right]}{4 b}- \\
-\frac{\mathrm{ae}^{-\mathrm{a}\left(\mathrm{t}-\mathrm{t}_{\mathrm{w}}\right)}\left[\cos \left(\mathrm{bt}_{\mathrm{w}}\right) \sin (\mathrm{bt})-\mathrm{bt} \cos \left[\mathrm{b}\left(\mathrm{t}-\mathrm{t}_{\mathrm{w}}\right)\right]\right]}{2 b^{2}} .
\end{gathered}
$$

Rearranging terms, one can write the final form of the solution to Equation (18), which is given by

$$
X(\mathrm{t})=\frac{\mathrm{A}_{\text {pulse }}}{\mathrm{b}}\left[\mathrm{T}_{1}(\mathrm{t})-\mathrm{T}_{2}(\mathrm{t})\right] \Theta(t)
$$

\section{References}

[1] Kotzé, R.; Wiklund, J.; Haldenwang, R. Optimization of pulsed ultrasonic velocimetry system and transducer technology for industrial applications. Ultrasonics 2013, 53, 459469.

[2] Chen, Q.; Li, W.; Wu, J. Realization of a multipath ultrasonic gas flowmeter based on transit-time technique. Ultrasonics 2014, 54(1), 285-290.

[3] Raine, A. B.; Aslam, N.; Underwood, C. P.; Danaher, S. Development of an ultrasonic airflow measurement device for ducted air. Sensors 2015, 15, 10705-10722.

[4] Hamouda, A.; Manck, O.; Hafiane, M. L.; Bouguechal, N. -E. An enhanced technique for ultrasonic flow metering featuring very low jitter and offset. Sensors 2016, 16, 1008. 
[5] Dumoulin, C.; Deraemaeker, A. Design optimization of embedded ultrasonic transducers for concrete structures assessment. Ultrasonics 2017, 79, 18-33.

[6] Lethiecq, M.; Levassort, F.; Certon, D.; Tran-Huu-Hue, L.P. Piezoelectric Transducer Design for Medical Diagnosis and NDE. In: Piezoelectric and Acoustic Materials for Transducer Applications. Springer, Boston, MA, 2008.

[7] Taghaddos, E.; Ma, T.; Zhong, H.; Zhou, Q.; Wan, M. X.; Safari, A. Fabrication and characterization of single-aperture $3.5 \mathrm{MHz}$ BNT-based ultrasonic transducer for therapeutic application. IEEE Trans. Ultrason. Ferroelectr. Freq. Control. DOI: 10.1109/TUFFC.2018.2793874.

[8] Coutinho, F. R.; Ofuchi, C. Y.; Ramos de Arruda, L. V.; Neves Jr, F.; Morales, R. E. M. A new method for ultrasound detection of interfacial position in gas-liquid two-phase flow. Sensors 2014, 14, 9093-9116.

[9] Lee, S. Q.; Youm, W. Enlarging the short range detection limit using input shaping technique in low damping ultrasonic transducers. Proc. of the 24th Int. Congress on Sound and Vibr. 2017, London, UK.

[10] Gallego-Juárez, J. A. Piezoelectric ceramics and ultrasonic transducers. J. Phys. E: Sci. Intrsum. 1989, 22, 804-816.

[11] Yang, Y.; Wei, X.; Zhang, L.; Yao, W. The effect of electrical impedance matching on the electromechanical characteristics of sandwiched piezoelectric ultrasonic transducers. Sensors 2017, 17, 2832.

[12] Shrout, T. R.; Zhang, S. J. Lead-free piezoelectric ceramics: Alternatives for PZT? J. Electroceram. 2007, 19, 111-124.

[13] Aksel, E.; Jones, J. L. Advances in lead-free piezoelectric materials for sensors and actuators. Sensors 2010, 10, 1935-1954. 
[14] Rödel, J.; Webber, K. G.; Dittmer, R.; Jo, W.; Kimura, M.; Damjanovic, D. Transferring lead-free piezoelectric ceramics into application. J. Eur. Ceram. Soc. 2015, 35, 16591681.

[15] Lee, S. T. F.; Lam, K. H.; Zhang, X. M.; Chan, H. L. W. High-frequency ultrasonic transducer based on lead-free BSZT piezoceramics. Ultrasonics 2011, 51, 811-814.

[16] Fan, F.; Zhao, X.; Wang, F.; Yue, Q.; Zhou, H.; Luo, H.; Tang, Y.; Duan, Z.; Shi, W.; Fang, B. Design and fabrication of high frequency ultrasonic transducer based on lead-

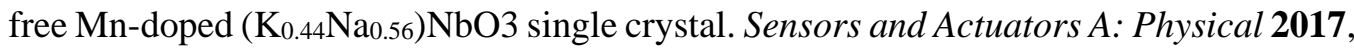
267, 182-186.

[17] Hong, C.-H.; Kim, H.-P.; Choi, B.-Y.; Han, H.-S.; Son, J.; Ahn, C.; Jo, W. Lead-free piezoceramics -- Where to move on? J Materiomics 2016, 2, 1-24.

[18] Yang, Z.; Zu, J. Comparison of PZN-PT, PMN-PT single crystals and PZT ceramic for vibration energy harvesting. Energy Conversion and Management 2016, 122, 321-329.

[19] Tohmyoh, H. Polymer acoustic matching layer for broadband ultrasonic applications. $J$. Acoust. Soc. Am. 2006, 120 (1), 31-34.

[20] Callens, D.; Bruneel, C.; Assaad, J. Matching ultrasonic transducer using two matching layers where one of them is glue. NDT\&E Int. 2004, 37, 591-596.

[21] Trogé, A.; O'Leary, R. L.; Hayward, G.; Pethrick, R. A.; Mullholland, A. J. Properties of photocured epoxy resin materials for application in piezoelectric ultrasonic transducer matching layers. J. Acoust. Soc. Am. 2010, 128 (5), 2704-2714.

[22] Hotate, M.; Yoshidome, D.; Kojima, T.; Hoshina, T.; Takeda, H.; Tsurumi, T. Design and fabrication of acoustic matching layer for lead-free ultrasonic flowmeter. J. Chem. Soc. Jpn. 2015, 123, 321-327. 
[23] Zhou, Q.; Lam, K. H.; Zheng, H.; Qiu, W.; Shung, K. K. Piezoelectric single crystal ultrasonic transducers for biomedical applications. Progress in Materials Science 2014, $66,87-111$.

[24] Tomikawa, Y.; Yamada, H.; Onoe, M. Wide band ultrasonic transducer using tapered piezoelectric ceramics for non-destructive inspection. Jpn. J. Appl. Phys. 1984, 23, 113115.

[25] Rybyanets, A. N.; Naumenko, A. A.; Sapozhnikov, O. A.; Khokhlova, V. A. New methods and transducer designs for ultrasonic diagnostics and therapy. Phys. Procedia 2015, 70, 1152-1156.

[26] de León, B. A.; Mossberg, M. Identification of under-damped second-order systems using finite duration rectangular pulse inputs. Proc. of the 2007 Am. Control Conf. 2007, 14244-0989-6.

[27] Smith, S. W. The Scientist and Engineer's Guide to Digital Signal Processing, Newnes, 2002.

[28] Güler, I.; Güler, N. F. Construction and matching of ultrasonic transducer for pulsed Doppler blood flowmeter. Proc. Ann. Inter. Conf. of the IEEE. Eng. Med. Biol. Soc. 1990, 12 (1), 320-321.

[29] Altiparmak, D.; Güler, I. Construction and testing of ultrasound broadband transducers for blood flow sensing. Journal of Medical Systems 1998, 22, 278-285.

[30] Arnold, F. J.; Gonçalvez, M. S.; Roger, L. B.; Müllen, S. S. Electrical impedance of piezoelectric ceramics under acoustic loads. ECTI Trans. on Electr. Eng. Electr. Comm. 2014, 12 (2), 48-54.

[31] Zemanek, J. Beam behavior within the nearfield of a vibrating piston. J. Acoust. Soc. Am. 1971, 49 (1), 181-191. 
This is the post-print (i.e. final draft post-refereeing) of the publication.

The final publication is available at Taylor \& Francis Group via http:dx.doi.org/10.1080/10739149.2018.1518876

[32] Ullate, L. G.; San Emertio, J. L. A new algorithm to calculate the transient near-field of ultrasonic phased arrays. IEEE Trans. Ultrason. Ferroelectr. Freq. Control 1992, 39 (6), 745-753.

[33] Shung, K. K.; Zipparo, M. Ultrasonic transducers and arrays. IEEE Eng. Med. Bio. 1996, 15 (6), 20-30. 


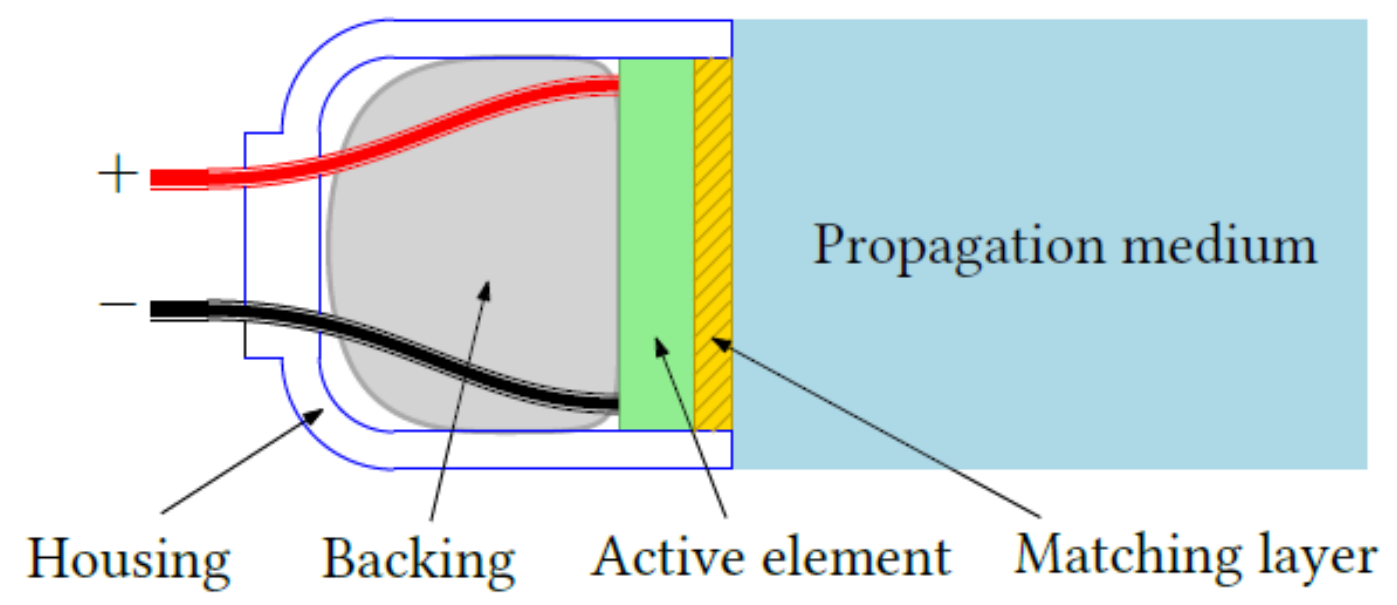

Figure 1: Sketch of an ultrasonic immersion transducer for general purposes. The main components of an ultrasonic transducer are the active element, matching layer, backing and housing. 

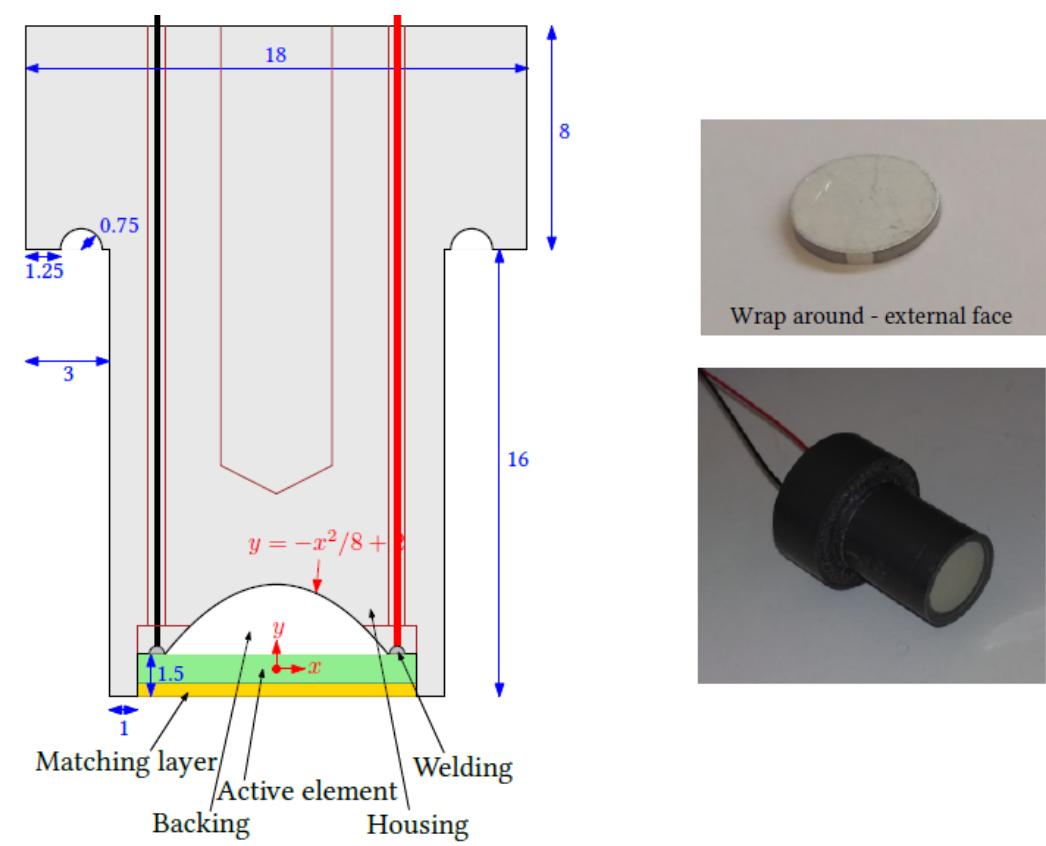

Wrap around - external face
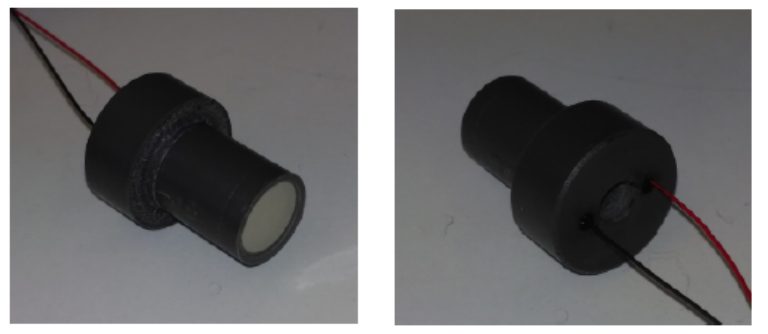

Figure 2: Left: schematics of the geometry of the housing. The paraboloid shape in the rear face of the backing is designed with the aim to maximize the transmission to the propagation medium. Two holes have been allocated in the back face of the active element to give space for the welding. Right: Pictures of the active element with wrap-around electrodes and the assembled transducer. 


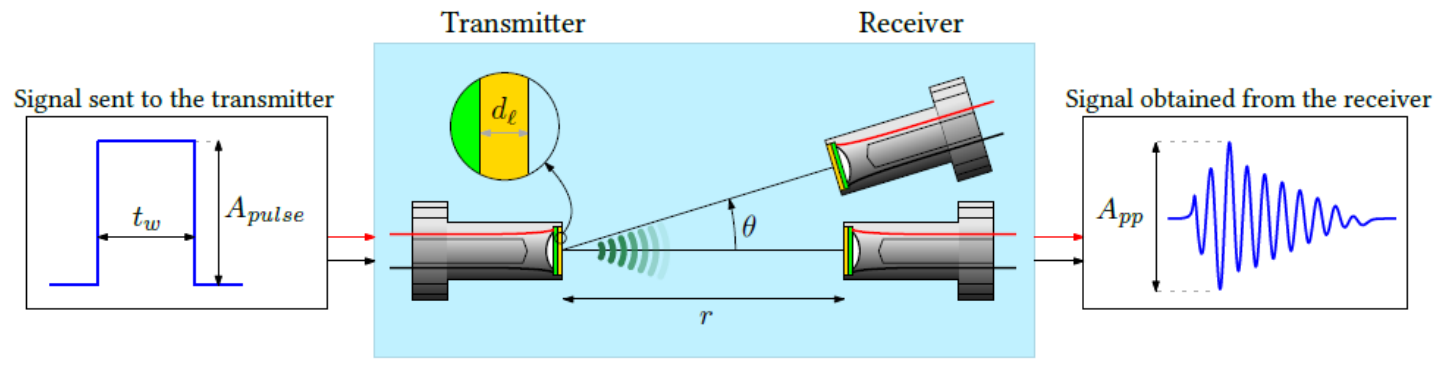

Figure 3: Schematics of the measurement setup configuration and key parameters used in this section. Two identical transducers are placed face to face, separated by a distance $r$ and forming an incidence angle $\theta$. A rectangular pulse with amplitude $A_{p u l s e}$ and a duration $t_{w}$ is sent to the transmitter, which converts the electric pulse into acoustic waves that propagate in water. The receiver transforms the ultrasonic waves back to an electric signal with a certain peak-to-peak amplitude $A_{p p}$. The thickness of the matching layer, in both transducers, is $d_{\ell}$. 


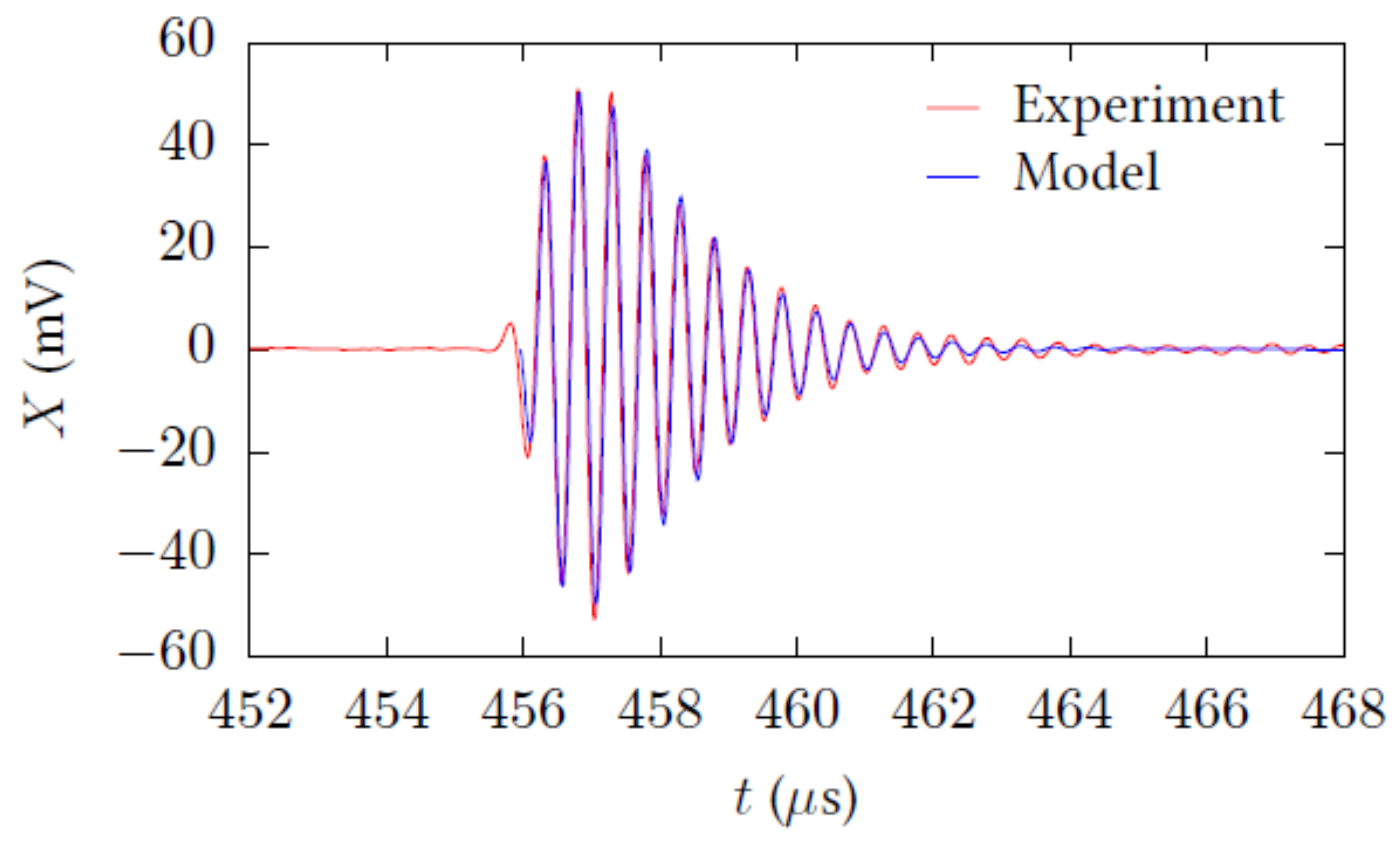

Figure 4: Amplitude of the electric signal received at the target transducer as a function of time. Red line corresponds to the received signal obtained experimentally, and filtered by Equation 15 with $N_{\mathrm{MA}}=100$. Black line shows the prediction by the analytical solution (Equation (14)). A comparison between the experimentally received signal and the prediction by the proposed model indicates a high degree of similarity between the two curves. 

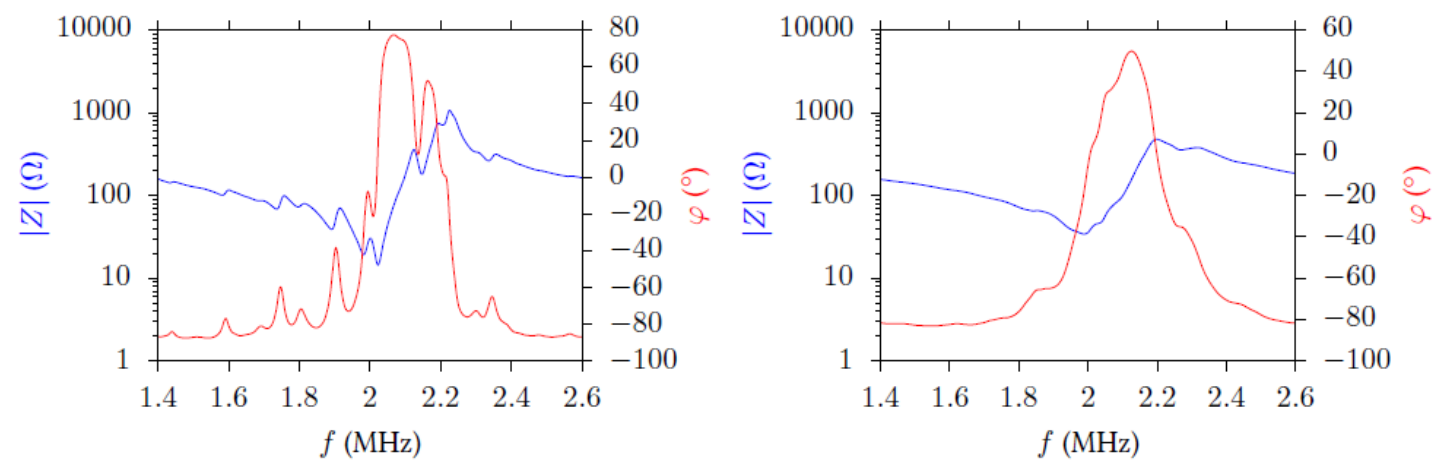

Figure 5: Electric impedance and phase as a function of the frequency. Blue line: electric impedance; red line: phase. Minima in electric impedance with an abrupt phase change correspond to resonance modes, maxima in electric impedance correspond to anti-resonance modes. 

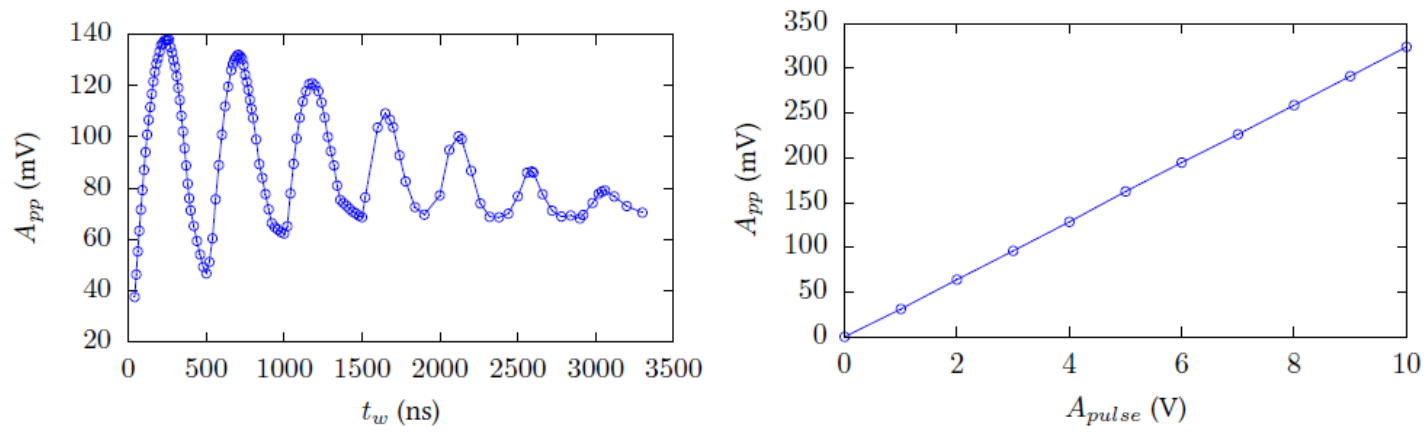

Figure 6: Left: Peak-to-peak amplitude of the received signal as a function of the pulse duration.

The position of maxima and minima of energy transmission is predicted by Equation (16). Right:

Peak-to-peak amplitude of the received signal as a function of the applied pulse amplitude. High linearity is achieved in the studied range of voltages. 

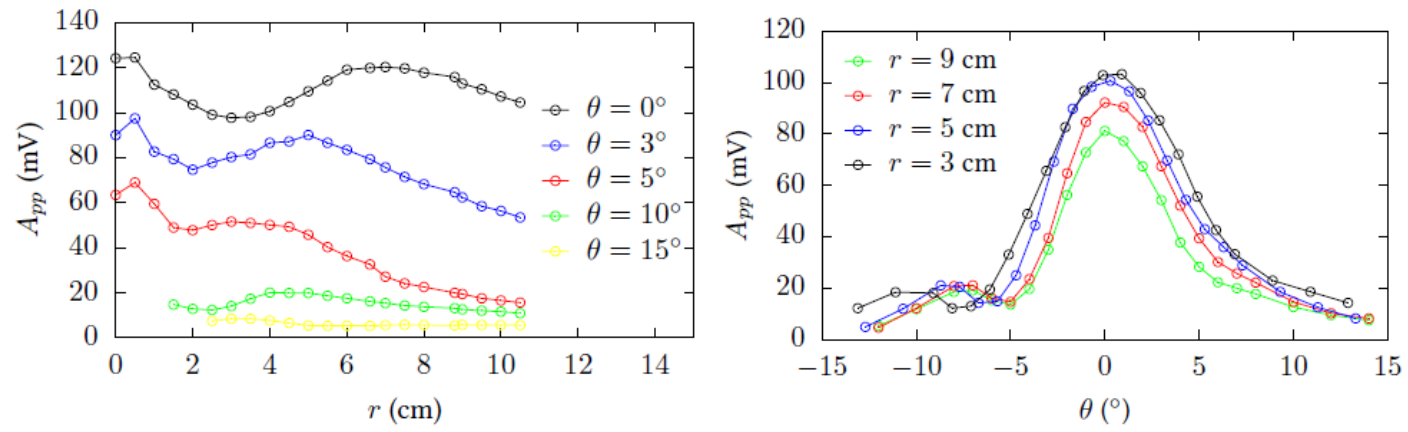

Figure 7: Left: Peak-to-peak amplitude of the received signal as a function of the distance between transducers. A slight decrease in amplitude is observed at short distances $(r<4 \mathrm{~cm})$, that could be due to the near field wave disturbances. Right: Peak-to-peak amplitude of the received signal as a function of the incidence angle. A significant decrease in amplitude for angles higher than a small critical value $\left(\theta c \approx 5^{\circ}\right)$ reveals that transducers are highly collimated. 


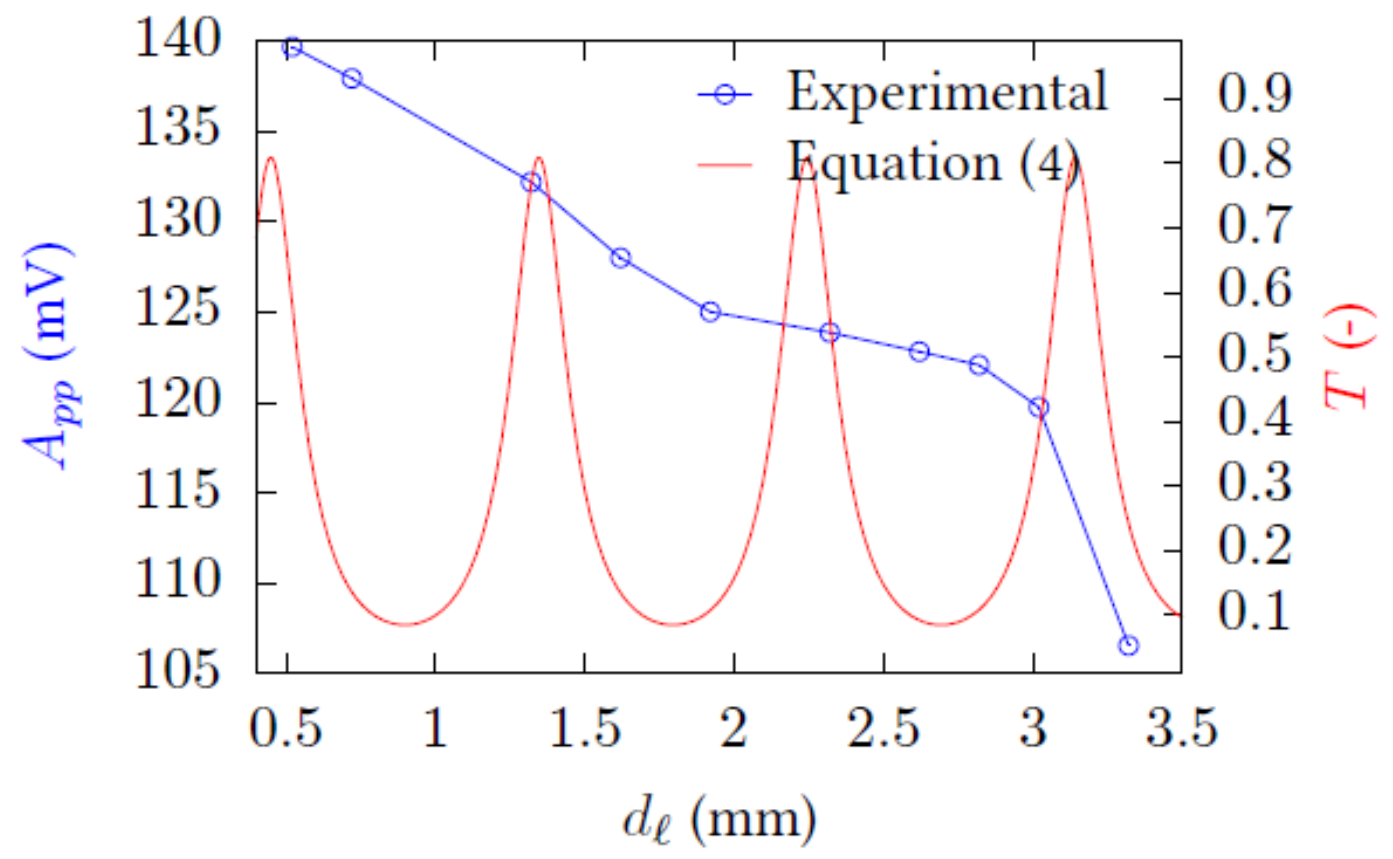

Figure 8: Peak-to-peak amplitude of the received signal as a function of the thickness of the matching layer, and theoretical transmission coefficient. Smooth decrease in amplitude is observed as the thickness increases. This behavior is not predicted by Equation (4). 